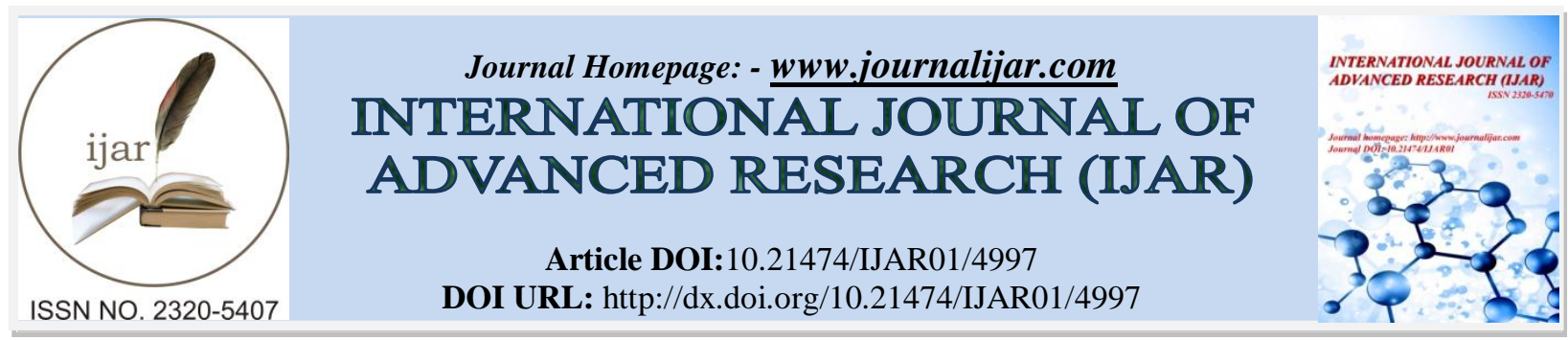

RESEARCH ARTICLE

\title{
THE NEWTONIAN MODEL OF THE GRAVITATIONAL RED SHIFT AND THE MEASURE OF THE DISTANCES IN COSMOLOGY.
}

\author{
AnathnathGhosh.
}

Dum Dum Motijheel College Motijheel Avenue, Kolkata West Bengal, India.

\section{Manuscript Info}

Manuscript History

Received: 28 May 2017

Final Accepted: 30 June 2017

Published: July 2017

Key words:-

Gavitation, Red shift, Galaxy,

Distance Modulous, Cosmology.

\section{Abstract}

The measurement of the distances of the astrophysical objects is very ifficult task in cosmology. From experimental point of view, different cosmological ladders are used to measure the distances of the stars of the galaxies with the help of the redshift calculations of the astrophysical objects. As we know that the gravitational redshift plays an important role in this regard because it carries the information of the potential field at which the photon is emitted and the application of the Newtonian gravity to calculate the distances of the white dwarf and neutron stars are possible provided we know their masses. In spectroscopy the measurement of the redshift can be done very accurately. The theoretical calculation of the gravitational red shift requires the combination of Newtonian gravity, particle theory of light and weak equivalence principle of gravitating mass equals inertial mass. According to this theory, the light emitted by the distance stars get stretched and this phenomenon is known as gravitational red shift. The wavelength of the light emitted by the stars is increased and consequently the frequency of the light received by us on earth is decreased. In this paper, we use Newtonian gravity and the property of the homogenous and isotropic space to estimate the various parameters of the astrophysical objects by the theoretical study of the gravitational redshift and compare them with the experimental results.

Copy Right, IJAR, 2017,. All rights reserved.

\section{Introduction:-}

The discovery of accelerating universe by studying the SNeIa supernova explosion data is remarkable in relation to the experimental achievements in cosmology. The explanation relating to these path breaking observations assume the existence of a hitherto unknown dark energy and dark matter[3]. Einstein general theory of relativity along with the cosmological constant representing the dark energy of the $\Lambda \mathrm{CDM}$ model is believed to be responsible for the accelerated state of the universe. But the challenges of this model are to prove and explain the existence of dark matter and dark energy of the universe. So far there is no proof of the existence of the dark matter or dark energy and according to the present author, the gravitational redshift is a key parameter in the study of the cosmological model because from the experimental point of view, it can be measured accurately by the spectroscopic method. Actually redshift is generally related with the velocity of the object. The theory of the gravitational red shift predicts that the wavelength of electromagnetic waves is lengthened when it passes from a lower gravitational potential well to a higher potential level. As the velocity of the electromagnetic waves remains same, the energy of the photons 
will decrease and hence the frequency of the photons decreases. Therefore, the wav7elength of the photons increases. For example, if a photon of energy $h \frac{c}{\lambda}$, where $\mathrm{h}$ is the planck constant, $\mathrm{c}$ is the velocity of light and $\lambda_{0}$ is the wavelength of the photon when it is emitted from the surface of the stars, then the increase of the wavelength of the photon or shift of the wavelength towards the red end of the electromagnetic spectrum is known as the gravitational red shift. The gravitational red shift was confirmed experimentally first by Robert Pound and his graduate student Glen. A. Rebka Jr. in 1959[2]. It is a gravitational red shift experiment, which measures the redshift of light moving in a gravitational field. It is considered to be the experiment that ushered in an era of precession measurements of cosmological parameters[3-10]. The converse is also true. The observed wavelength of a photon falling into a gravitational well will be shortened, or gravitationally 'blueshifted', as it gains energy.

According to the cosmological principle, the universe is thought to be isotropic and homogenous when looked at it from any point of observation in the universe. So we can think the observable universe to be a homogenous sphere of radius equivalent to the Hubble radius $\mathrm{R}(\mathrm{c} / \mathrm{H})$, where $\mathrm{c}$ is the velocity of light and $\mathrm{H}$ is the Hubble constant. If we consider a mass inside this sphere of radius $\mathrm{R}$, then according to the Newtonian mechanics, the potential contribution on it will come from two parts. One part is due to the inner sphere of radius say $r$, and the other part will come from the spherical shell of radii between R and Rs, where Rs is the sphere of photon emitting star. Here we consider the universe centered around the star, and the observer is situated at a large distance $r$ on earth from the star.

The situation is similar to the case of the quantum mechanical description of an electron[1] bound to an atom in an excited state. When a revolving electron in an excited state jumps from a higher energy state to a lower energy stats it emits photon with frequency corresponding to the difference of the energy states. In the reverse case, when an electron goes up to a higher energy state from a lower energy state, it absorbs a photon of energy appropriate to the difference of the energy between the states.

Now consider two copies of this electron-atom system, one in the excited state (the emitter), the other in the lower energy state (the receiver). If the two systems are stationary relative to one another and the space between them is flat (i.e. we neglect gravitational fields) then the photon emitted by the emitter can be absorbed by the electron in the receiver. However, if the two systems are in a gravitational field then the photon may undergo gravitational redshift as it travels from the first system to the second, causing the photon frequency observed by the receiver to be different to the frequency observed by the emitter when it was originally emitted. Another possible source of redshift is the Doppler shift. If the two systems are not stationary relative to one another then the photon frequency will be modified by the relative speed between them.

In the Pound-Rebkaexperiment[2], the emitter was placed at the top of tower with the receiver at the bottom. The gravitational field of the Earth will cause a photon emitted downwards (towards the Earth) to be blueshifted (i.e. its frequency will increase). To counteract the effect of gravitational redshift, the emitter was moved upwards (away from the receiver) causing the photon frequency to be red shifted, according to the Doppler shift. In this paper, Newtonian mechanics is applied to calculate the gravitational redshift of a star and show how accurately we can determine the distance of the stars which is a very difficult task in cosmology. The supernova type Ia explosion has characteristic light curves which are the plot of the luminosity versus time after the explosion of the stars. The luminosity curves indicate the presence of intermediate elements characteristic spectra corresponding to the mass ranging from oxygen to calcium forming the outer layesr of the stars. After several months of the star's explosion, the outer layers of the stars fade away to the point of transparency and the characteristic spectra corresponding to the core layers of the stars formed mostly by iron dominate the energy release of the stars . The use of type Ia supernovae to measure cosmological distances precisely was done by the pioneering works of the collaboration of the Chilean and US astronomers. Their study shows that the peak luminosity of the light curves of the stars do not reach the same peak value and a single parameter measured from the curves of the type Ia supernovae[10-16] can be used to correct standard candle values. Here we also compare with their results, the distance modulous versus redshift curves which qualitatively are consistent with the observed experimental results. The Newtonian mechanics is quite able to explain the observed experimental results so far without the assumption of the dark matter and dark energy. 


\section{Theory and formula:-}

According to the Hubble law, the galaxies are moving away from each other following the relation , $\mathrm{v}=\mathrm{H} \mathrm{r}$ where $\mathrm{v}$ is the relative velocity between the galaxies, $\mathrm{r}$ is the separation among them and $\mathrm{H}$ is the Hubble constant. So the limit $\mathrm{r}$ of the observable universe should be $\mathrm{c} / \mathrm{H}$ when the maximum relative velocity of the galaxies is of the order of the velocity of light $\mathrm{c}$. We cannot establish the causal contact more than the finite distance $\mathrm{c} / \mathrm{H}$.

So the classical Newtonian mechanics gives the expression for the gravitational potential inside a solid sphere at a distance $\mathrm{r}$ from the center $\mathrm{O}$ is as shown in Fig.1. From the figure we can write the energy conservation formula for a photon being emitted in a gravitational field and received at a distance in different gravitational field as,

$h v_{0}-\frac{G M_{u}}{2 c^{2} R^{3}}\left(3 R^{2}-r^{2}\right) h v_{0}=h v_{e}-\frac{G M_{u}}{2 c^{2} R^{3}}\left(3 R^{2}-R_{s}^{2}\right) h v_{e}$

Where $v_{0}$ and $v_{e}$ are respectively the observed and emitted photon frequency and $M_{s}$ and $M_{u}$ are the masses of the photon emitting star and the universe respectively. $R_{S}$ is the radius of the emitter of the photon, $R$ is the radius of the observable universe and $r$ is the distance between the center of the star and the point at which the observer on the earth is situated. From the above formula we can calculate the gravitational redshift as

$1+z=1-\frac{G M_{u}}{2 c^{2} R^{3}}\left(3 R^{2}-r^{2}\right) /\left(1-\frac{G M_{u}}{2 c^{2} R^{3}}\left(3 R^{2}-R_{S}^{2}\right)\right)$

or alternatively we can calculate the distance of the star from us as,

$r=\left[z\left(1-\frac{G M_{u}}{2 c^{2} R}\right)\left(\frac{2 c^{2} R^{3}}{G M_{u}}\right)\right]^{\frac{1}{2}}$

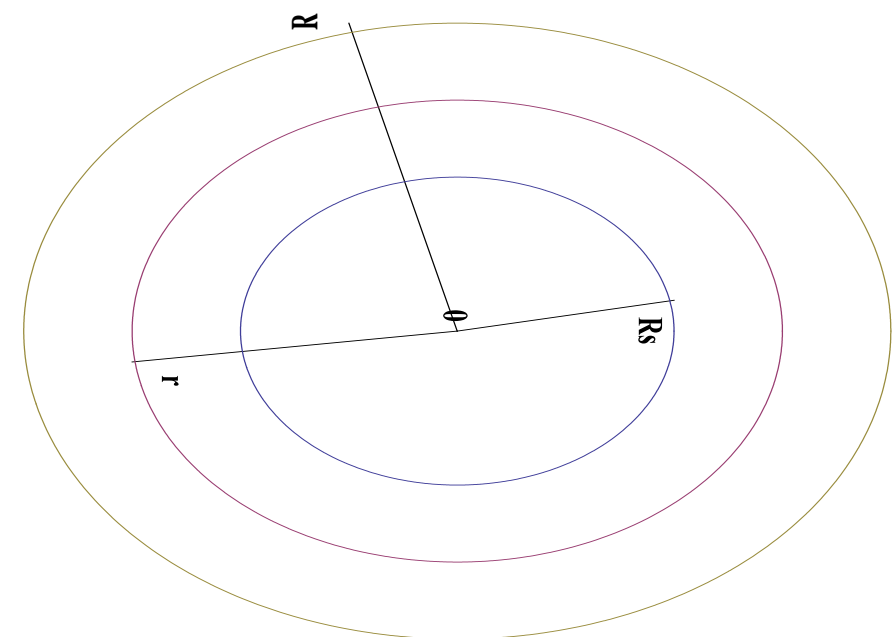

Fig.1:-The central sphere represents the star itself. The outer sphere is the sphere of Hubble radius $(\mathrm{c} / \mathrm{H})$. The potential inside point of a sphere at a distance $r$ is shown.

If the condition for $1-\frac{G M_{u}}{2 c^{2} R^{3}}\left(3 R^{2}-R_{S}^{2}\right)=0$ is satisfied, then there will be no emission of photon of the stars, and for this condition we get

$$
1 \sim \frac{3 G M_{u}}{2 c^{2} R}=\frac{2 \pi G R^{2} \rho}{c^{2}}
$$

where $\rho$ is the density of the universe at this point of the star. So the relation $\rho R^{2}=\frac{c^{2}}{2 \pi G}$ is a constant which can be used to explain the flat rotation curves of the stars of spiral galaxy (see Fig.5). 


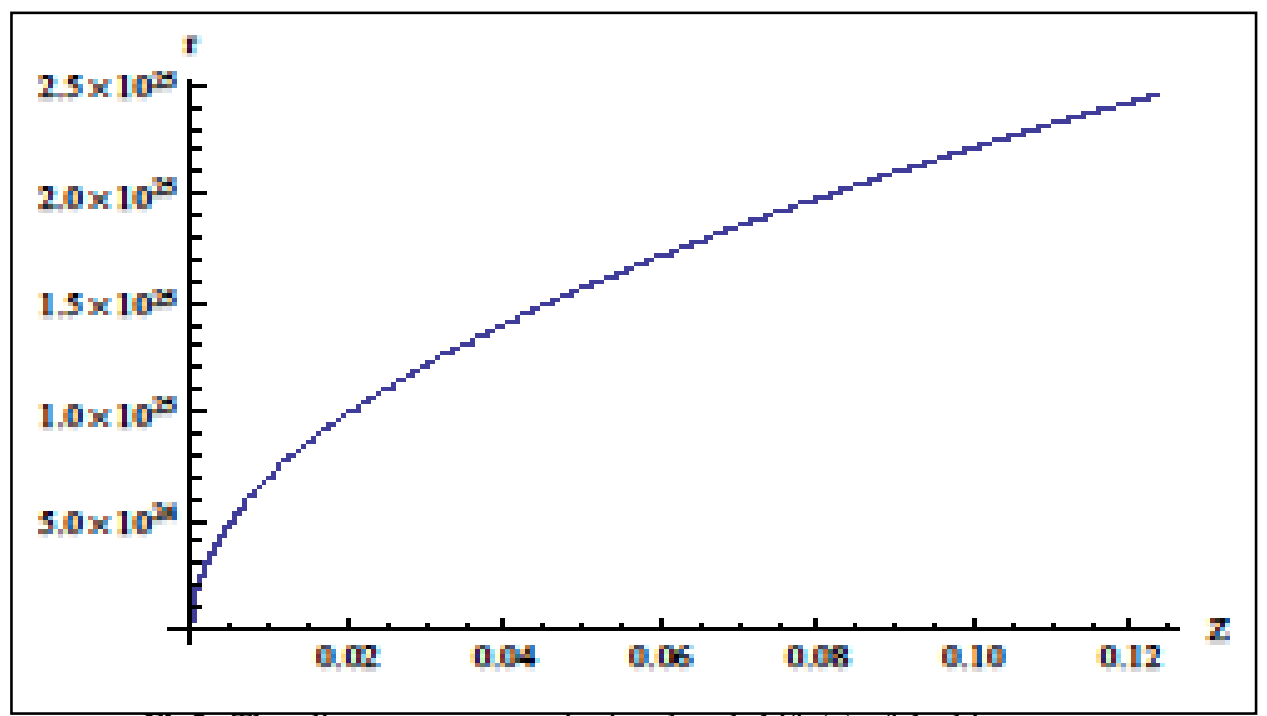

Fig2:-The distance r vs. gravitational red shift (z) of the binary stars.

We can calculate the masses of the binary neutron stars in the following method given below. If two binary stars of masses $M_{1}$ and $M_{2}$ orbit around their common centre of mass as in Fig.3

Then according to the definition of the centre of mass we can write,

$M_{1} a_{1}=M_{2} a_{2}$

Where $\mathrm{a}$ is the total separation between the binary stars and

or $a_{2}=$

$a=a_{1}+a_{2}$ (5)

$M_{1} \frac{a}{M_{1}+M_{2}}$.

From Newton's third law for circular motion to $M_{2}$, we get

$G \frac{M_{1} M_{2}}{a^{2}}=M_{2} a_{2} \Omega^{2}$, where $\Omega$ is the angular velocity of the binary. Substituting for $a_{2}$ from equation (6) we get,

$\Omega=\sqrt{\frac{G\left(M_{1}+M_{2}\right)}{a^{3}}}$

$P=\frac{2 \pi}{\Omega}$

$P^{2}=4 \frac{\pi^{2}}{G\left(M_{1}+M_{2}\right)} a^{3}$

For visual binary, looking the orbits of each stars we can determine the ratio of the masses $\frac{M_{1}}{M_{2}}$. From knowing the angular separation of the stars, the sum of the masses $M_{1}+M_{2}$ can be determined .

Now consider spectroscopic binaries with circular orbits. The velocities are constant around the orbits. 


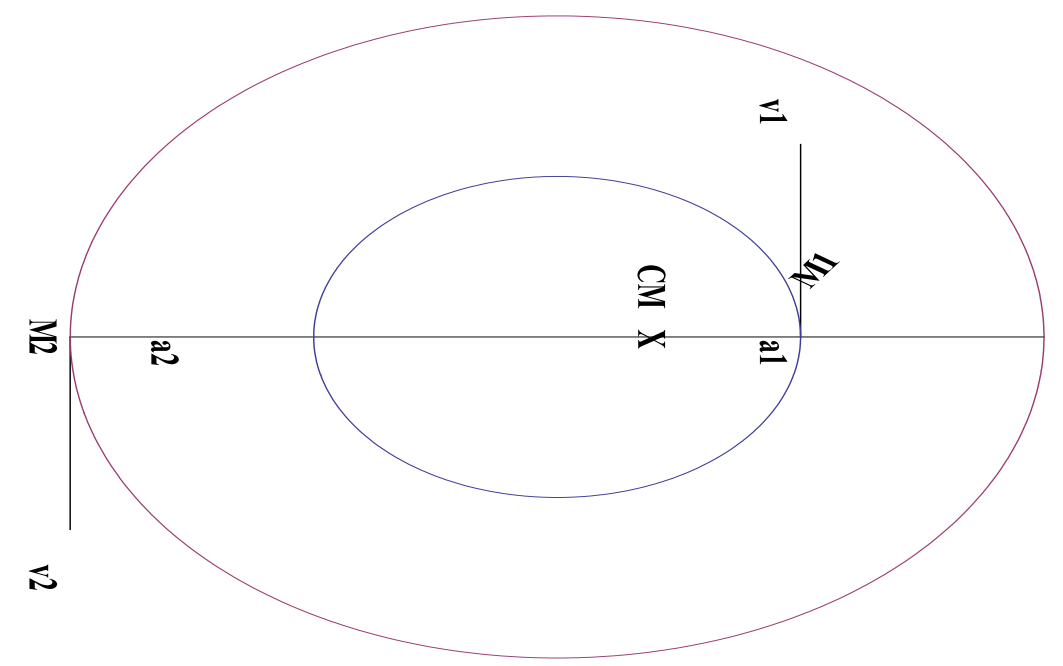

Fig.3:- The circular orbits of the binary stars of masses $M_{1}$ and $M_{2}$.

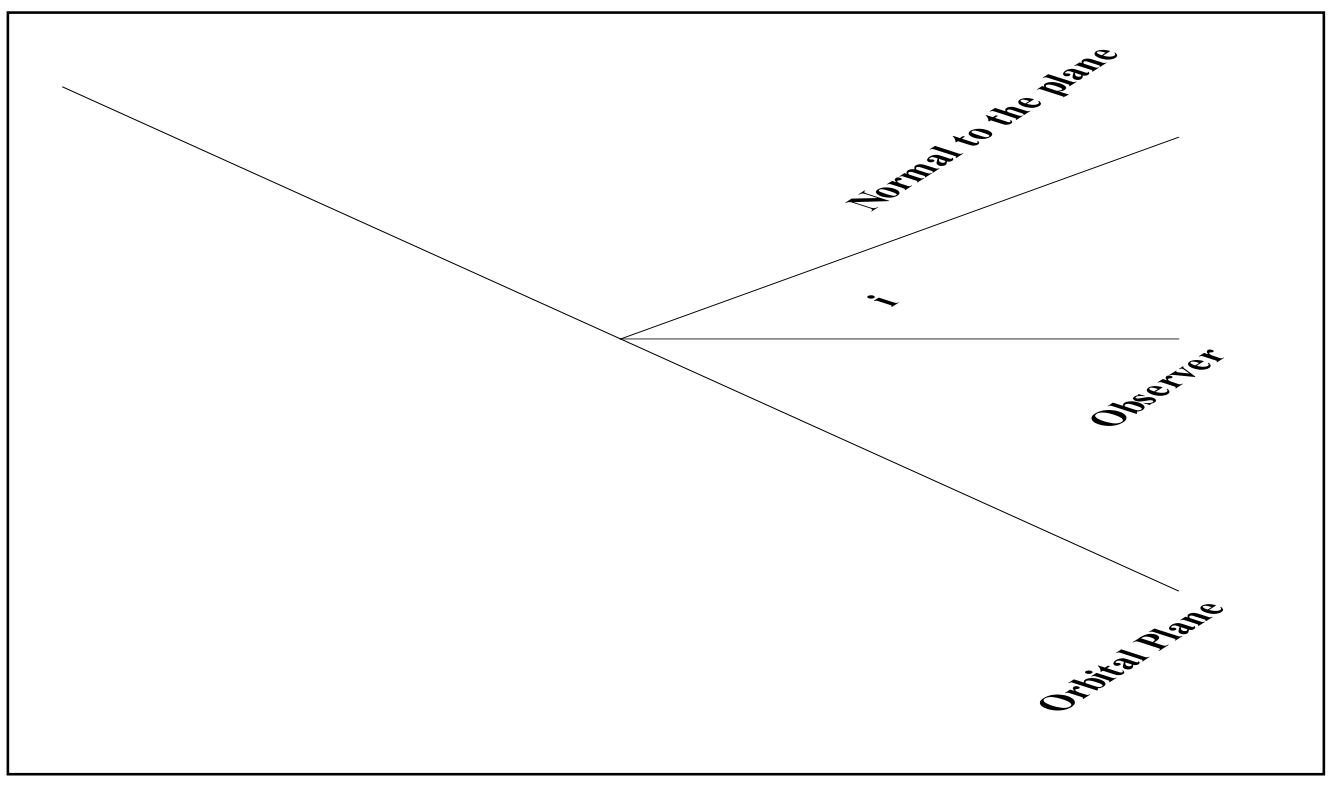

Fig.4 The plane of the orbits of the binary stars and the direction of observation.

For the circular orbit, we can write for the two masses,

$P v_{1}=2 \pi a_{1} a n d P v_{2}=2 \pi a_{2}$, but if the plane of the orbit is inclined to an angle $\mathrm{i}$ as shown in fig.4, the expressions for the relative velocities are,

$v_{r 1}=v_{1} \sin i$ and $v_{r 2}=v_{2} \sin i$

And

therefore, from Kepler's third law we can write,

$\mathrm{M}_{1}+\mathrm{M}_{2}=\frac{\mathrm{P}\left(\mathrm{v}_{1}+\mathrm{v}_{2}\right)^{3}}{2 \pi \mathrm{G}}=\frac{\mathrm{P}\left(\mathrm{v}_{\mathrm{r} 1}+\mathrm{v}_{\mathrm{r} 2}\right)^{3}}{2 \pi \mathrm{G} \sin ^{3} \mathrm{i}}$,

Where $\mathrm{i}$ is the inclination of the orbit. The detailed study of the light curves gives the angle of the inclination angle. Knowing $\mathrm{P}, v_{1} a n d v_{2}$ we can find the masses of the binary stars using equations (4) -(11). Ratio of the observed radial velocities is 


$$
\frac{v_{r 2}}{v_{r 1}}=v_{2} \sin i / v_{1} \sin i=\frac{2 \pi a_{2} / P}{2 \pi a_{1 / P}}=\frac{a_{2}}{a_{1}} .
$$

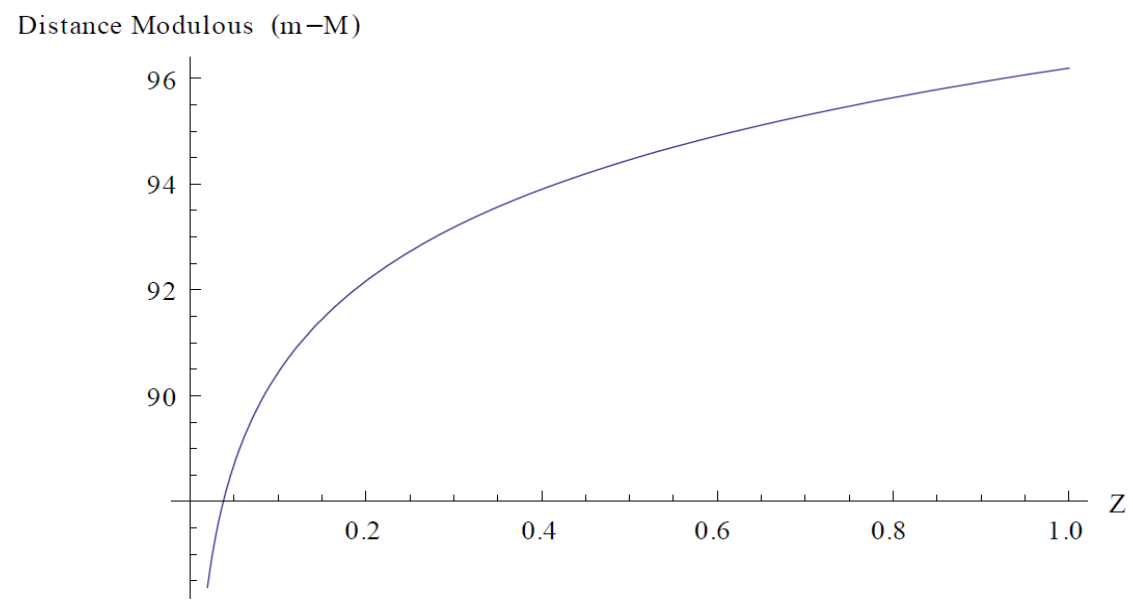

Fig.5:- The distance modulous (m-M) vs. red shift $(\mathrm{Z})$ of stars from the centre.

\section{Results and Discussions:-}

In this paper, we have calculated the distance of the stars from the earth as a function of the gravitational redshift (z). The nature of the function ' $r$ ' is plotted against $\mathrm{z}$ and it has been shown in Fig.2. It is to be observed that the figure has remarkable similarity with the commoving distance of the $\Lambda \mathrm{CDM}$ model[ 17]. It is well known that the rotation curves of the stars of the spiral galaxy are flat. We have calculated the velocity of the stars as a function of the distances from the centre of the galaxies. The curves qualitatively resembles the observed flat rotation curves. Another interesting features of the stars are the distance modulous (m-M) vs. redshift graph. It is quite interesting that the curves (Fig.6) are similar to that observed by the authors

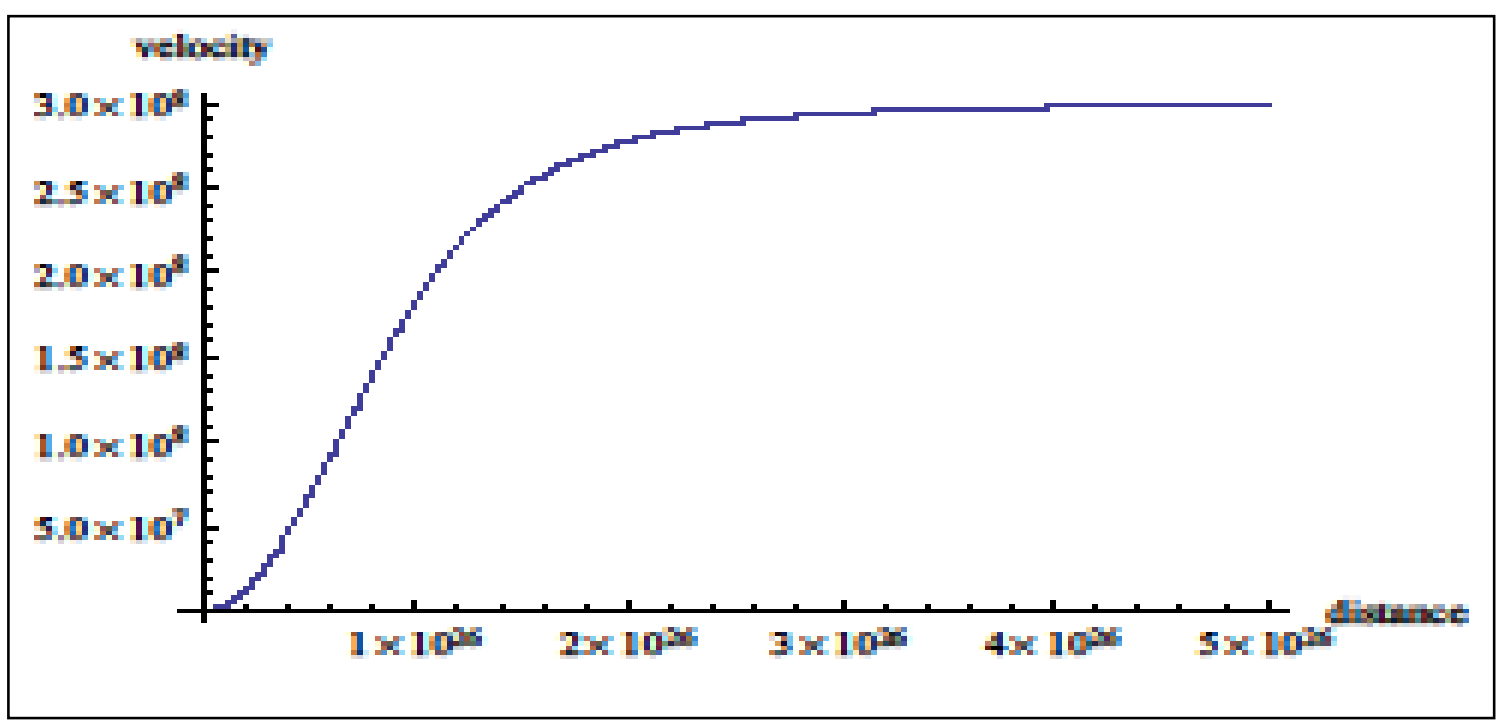

Fig. 6:-The velocity V vs. distance of the stars from the centre.

Perlmutter, S. et al[11]. Also we have observed that the product of the critical density and radius of the observable universe satisfies the relation

$$
\rho R^{2}=\frac{c^{2}}{2 \pi G}=2.1465510^{26} \frac{\mathrm{kg}}{\mathrm{m}}
$$


Now if we take the standard value of $\mathrm{R}$ as $R=10^{26} \mathrm{~m}$ then we can find the density of the universe of the order of $10^{-26} \frac{\mathrm{kg}}{\mathrm{m}^{3}}$ and the mass of the universe comes out to be of the order of $10^{53} \mathrm{~kg}$.

\section{Conclusions:-}

The isotropic and homogenous space in cosmological scales is exploited to calculate the various parameters in cosmology. The galaxy rotation curves agree qualitatively with the observed data. The distance modulous graphs of stars agree the experimental data without any assumptions of dark energy or dark matter. The qualitative features of all theoretical observations in the study of the models of the cosmology are quite interesting and deserves special attention. This investigation is particularly useful to study the properties of the white dwarf and the binary stars. In case of the binary stars the masses can be measured very accurately and hence the use of the gravitational redshift is helpful to estimate the distances in cosmology.

\section{Acknowledgements:-}

I am grateful to Dr. Subhendu Rajbanshi for his help in making some of the graphics.

\section{References:-}

1. Pound, R. V.; Rebka Jr. G. A. (November 1, 1959). "Gravitational Red-Shift in nuclear Resonance". Physical Review Letters. 3 (9): 439-441.

2. Bahcall, N., Ostriker, J., Perlmutter, S., and Steinhardt, P. 1999, Science, 284, 148

3. Balbi, A., et al. 2000, Astrophysical Journal Letters, 545, 1

4. 4. Carroll, S., Press, W., and Turner, E., 1992, Annual Review of Astronomy and Astrophysics, 30, 499

5. de Bernardis, et al., 2000, Nature, 404, 955

6. Guth, A.1997, The inflationary universe. The quest for a new of cosmic origins, Reading: AddisonWesleyHogan, C., Kirshner, R., and Suntzeff, N. 1999, Scientific American, 280, 28

7. Leibundgut, B. 2000, The Astronomy and Astrophysics Review, 10, 179

8. Ostriker, J. and Steinhardt, P.. 2001, Scientific American, 284, 36

9. Peebles, J. 2001, Scientific American, 284, 44

10. Perlmutter, S., et al. 1999, Astrophysical Journal, 517, 565

11. Riess, A. 2000, Publications of the Astronomical Society of the Pacific, 112, 1284

12. Riess, A., et al. 1998, Astronomical Journal, 116, 1009

13. 14. Virginia Trimble, Martin Bartow, "Gravitational red shift and white dwarf stars" Einstein Online vo1.04 (2010) 1006

14. 15. S. Perlmutter et al., Ap. J. 483, 565 (1999)

15. 16. S. Perlmutter et al., Nature 391, 51 (1998)

16. 17. " Distance measures in Cosmology" by David W. Hogg, arXiv: astro-ph/9905116v4 16 Dec. 2000 